\title{
Secondary Protein Structure
}

National Cancer Institute

\section{Source}

National Cancer Institute. Secondary Protein Structure. NCI Thesaurus. Code C13302.

The specific 3-dimensional folded protein structure that is determined by the hydrogen bonds formed between amino acids in the polypeptide chain. 